\title{
Existence and uniqueness of Chern connection in the Klein-Grifone approach
}

\author{
Nabil L. Youssef ${ }^{1}$ and S. G. Elgendi ${ }^{2}$ \\ ${ }^{1}$ Department of Mathematics, Faculty of Science, \\ Cairo University, Giza, Egypt \\ ${ }^{2}$ Department of Mathematics, Faculty of Science, \\ Benha University, Benha, Egypt \\ E-mails: nlyoussef@sci.cu.edu.eg, nlyoussef2003@yahoo.fr \\ salah.ali@fsci.bu.edu.eg, salahelgendi@yahoo.com
}

\begin{abstract}
The Klein-Grifone approach to global Finsler geometry is adopted. A global existence and uniqueness theorem for Chern connection is formulated and proved. The torsion and curvature tensors of Chern connection are derived. Some properties and the Bianchi identities for this connection are investigated. A concise comparison between Berwald, Cartan and Chern connections is presented.

Keywords: Barthel connection, Berwald connection, Cartan Connection, Chern connection, torsion tensors, curvature tensors, Bianchi identities.
\end{abstract}

MSC 2010: 53C60, 53B40, 58B20, 53C12.

\section{Introduction}

The most well known and widely used approaches to global Finsler geometry are the Klein-Grifone (KG-) approach ([4], [5], [6]) and the pullback (PB-) approach ([1], 2], 7], [9]). Each of the two approaches has its own geometry which differs significantly from the geometry of the other (in spite of the existence of some links between them).

In the PB-approach, the existence and uniqueness theorems for the four fundamental linear connections (Berwald, Cartan, Chern and Hashiguchi connections) on a Finsler manifold $(M, E)$ have been satisfactorily established ([11], [12]). In the KGapproach, Grifone [5] has investigated Cartan and Berwald connections. Szilasi and Vincze [8] have studied Chern and Hasiguchi connections using the technique of lifting vector fields to the tangent bundle. To the best of our knowledge there is no proof, in the KG-approach, of the existence and uniqueness theorems for Chern and Hashiguchi connections.

In this paper, we formulate and prove an intrinsic existence and uniqueness theorem for Chern connection. We derive the torsion and curvature tensors of this connection. 
We also study the Bianchi identities and investigate the properties of the curvature tensors of Chern connection. Finally, an appendix concerning a global survey of Berwald, Cartan and Chern connections in the KG-approach is presented. It should finally be noted that the Frölicher-Nijenhuis formalism of vector forms [3] is used extensively in this work.

\section{Notation and Preliminaries}

In this section, we give a brief account of the basic concepts of the Klein-Grifone approach to global Finsler geometry $([4,[5],[6])$. The following notations will be used throughout this paper:

$M$ : a real differentiable manifold of finite dimension $n$ and class $C^{\infty}$,

$\mathfrak{F}(M)$ : the $\mathbb{R}$-algebra of differentiable functions on $M$, $\mathfrak{X}(M)$ : the $\mathfrak{F}(M)$-module of vector fields on $M$,

$\pi_{M}: T M \longrightarrow M$ : the tangent bundle of $M$,

$\pi: \mathcal{T} M \longrightarrow M:$ the subbundle of nonzero vectors tangent to $M$,

$V(T M)$ : the vertical subbundle of the bundle $T(T M)$,

$\pi^{-1}(T M):=\mathcal{T} M \times T M:$ the pullback bundle induced by $\pi$,

$i_{X}$ : the interior derivative with respect to $X \in \mathfrak{X}(M)$,

$d f$ : the exterior derivative of $f$,

$d_{L}:=\left[i_{L}, d\right], i_{L}$ being the interior derivative with respect to a vector form $L$,

$\mathcal{L}_{X}$ : the Lie derivative with respect to $X \in \mathfrak{X}(M)$.

We have the following short exact sequence of vector bundles

$$
0 \longrightarrow \pi^{-1}(T M) \stackrel{\gamma}{\longrightarrow} T(T M) \stackrel{\rho}{\longrightarrow} \pi^{-1}(T M) \longrightarrow 0,
$$

where the bundle morphisms $\rho$ and $\gamma$ are defined as usual. The vector 1-form $J$ on $T M$ defined by $J:=\gamma \circ \rho$ is called the natural almost-tangent structure of $T M$. The vertical vector field $C$ on $T M$ defined by $C:=\gamma \circ \bar{\eta}$, where $\bar{\eta}$ is the vector field on $\pi^{-1}(T M)$ given by $\bar{\eta}(u)=(u, u)$, is called the canonical or the Liouville vector field. One can show that the almost-tangent structure $J$ has the following properties:

$$
J^{2}=0, \quad[J, J]=0, \quad[C, J]=-J, \quad \operatorname{Im}(J)=\operatorname{Ker}(J)=V(T M) .
$$

A scalar $p$-form $\omega$ on $T M$ is semi-basic if $i_{J X} \omega=0, \forall X \in \mathfrak{X}(T M)$. A vector $\ell$-form $L$ on $T M$ is semi-basic if $J L=0$ and $i_{J X} L=0, \forall X \in \mathfrak{X}(T M)$.

A scalar $p$-form $\omega$ on $T M$ is homogeneous of degree $r$ if $\mathcal{L}_{C} \omega=r \omega$. A vector $\ell$-form $L$ on $T M$ is homogeneous of degree $r$ if $[C, L]=(r-1) L$.

A semi-spray on $M$ is a vector field $S$ on $T M, C^{\infty}$ on $\mathcal{T} M, C^{1}$ on $T M$, such that $J S=C$. A homogeneous semi-spray $S$ of degree $2([C, S]=S)$ is called a spray. If $S$ is a semi-spray, then

$$
J[J X, S]=J X, \forall X \in \mathfrak{X}(T M) .
$$

A nonlinear connection on $M$ is a vector 1-form $\Gamma$ on $T M, C^{\infty}$ on $\mathcal{T} M, C^{0}$ on $T M$, such that $J \Gamma=J, \Gamma J=-J$. The vertical and horizontal projectors $v$ and $h$ associated with $\Gamma$ are defined respectively by $v:=\frac{1}{2}(I-\Gamma), h:=\frac{1}{2}(I+\Gamma)$. Thus $\Gamma$ gives rise to the direct sum decomposition $T(T M)=V(T M) \oplus H(T M)$, where 
$V(T M):=\operatorname{Im} v=\operatorname{Ker} h$ is the vertical bundle and $H(T M):=\operatorname{Im} h=\operatorname{Ker} v$ is the horizontal bundle induced by $\Gamma$. An element of $V(T M)(\operatorname{resp} . H(T M))$ will be denoted by $v X($ resp. $h X)$. A nonlinear connection $\Gamma$ is homogeneous if $\Gamma$ is homogeneous of degree 1 as a vector form, i.e., $[C, \Gamma]=0$. For a homogeneous connection $\Gamma$, we have

$$
[C, h X]=h[C, X], \forall X \in \mathfrak{X}(T M) .
$$

The torsion $t$ of a nonlinear connection $\Gamma$ is the vector 2 -form on $T M$ defined by $t:=\frac{1}{2}[J, \Gamma]$. The curvature $\mathfrak{R}$ of $\Gamma$ is the vector 2 -form on $T M$ defined by $\mathfrak{R}:=-\frac{1}{2}[h, h]$. Given a nonlinear connection $\Gamma$, an almost-complex structure $F\left(F^{2}=-I\right)$ is defined by $F J=h$ and $F h=-J$. This $F$ defines an isomorphism of $T_{z}(T M)$ for all $z \in T M$.

Definition 2.1. A Finsler space is a pair $(M, E)$, where $M$ is a differentiable manifold of dimension $n$ and $E$ is a map $E: T M \longrightarrow \mathbb{R}$, called energy, satisfying the axioms:

(a) $E(u)>0$ for all $u \in \mathcal{T} M$ and $E(0)=0$,

(b) $E$ is $C^{\infty}$ on $\mathcal{T} M, C^{1}$ on $T M$,

(c) $E$ is homogeneous of degree 2: $\mathcal{L}_{C} E=2 E$,

(d) The exterior 2 -form $\Omega:=d d_{J} E$, called the fundamental form, has a maximal rank.

From now on, we will be placed on a Finsler space $(M, E)$.

Theorem 2.2. Let $(M, E)$ be a Finsler space. The vector field $S$ defined by $i_{S} \Omega=-d E$ is a spray, called the canonical spray.

Theorem 2.3. On a Finsler space $(M, E)$, there exists a unique conservative $\left(d_{h} E=0\right)$ homogeneous nonlinear connection $\Gamma$ with zero torsion. It is given by $\Gamma=[J, S]$, where $S$ is the canonical spray. Such a connection is called the canonical or Barthel connection associated with $(M, E)$.

Theorem 2.4. For a Finsler space $(M, E)$, there exists a unique linear connection $\stackrel{\circ}{D}$ on TM satisfying the following properties:
(a) $\stackrel{\circ}{D} J=0$,
(b) $\stackrel{\circ}{D} C=v$,
(c) $\stackrel{\circ}{D} \Gamma=0(\Longleftrightarrow \stackrel{\circ}{D} h=\stackrel{\circ}{D} v=0)$,
(d) $\stackrel{\circ}{D}_{J X} J Y=J[J X, Y]$,
(e) $\stackrel{\circ}{T}(J X, Y)=0$

$h$ and $v$ being the horizontal and vertical projectors of $\Gamma=[J, S]$ and $\stackrel{\circ}{T}$ is the (classicl) torsion of $\stackrel{\circ}{D}$. This connection is called the Berwald connection.

Berwald connection is completely determined by:

$$
\stackrel{\circ}{D}_{J X} J Y=J[J X, Y], \quad \stackrel{\circ}{D}_{h X} J Y=v[h X, J Y], \quad \stackrel{\circ}{D} F=0 .
$$

The (h)h-torsion $\stackrel{\circ}{T}(h X, h Y)$ of Berwald connection is given by $\stackrel{\circ}{T}(h X, h Y)=\mathfrak{R}(X, Y)$.

A metric $g$ can be defined on $T M$ by

$$
g(X, Y)=\Omega(X, F Y), \forall X, Y \in \mathfrak{X}(T M),
$$

where $F$ is the almost-complex structure associated with Barthel connection. 
Theorem 2.5. For a Finsler space $(M, E)$, there exists a unique linear connection $D$ on TM satisfying the following properties:
(a) $D J=0$,
(b) $D C=v$,
(c) $D \Gamma=0$,
(d) $D g=0$,
(e) $T(J X, J Y)=0$
(f) $J T(h X, h Y)=0$.

This connection is called the Cartan connection.

Cartan connection is completely determined by:

$$
D_{J X} J Y=\stackrel{\circ}{D}_{J X} J Y+\mathcal{C}(X, Y), \quad D_{h X} J Y=\stackrel{\circ}{D}_{h X} J Y+\mathcal{C}^{\prime}(X, Y), \quad D F=0,
$$

where $\mathcal{C}$ and $\mathcal{C}^{\prime}$ are the vector 2 -forms on $T M$ defined respectively by

$$
\Omega(\mathcal{C}(X, Y), Z)=\frac{1}{2}\left(\mathcal{L}_{J X}\left(J^{*} g\right)\right)(Y, Z), \quad \Omega\left(\mathcal{C}^{\prime}(X, Y), Z\right)=\frac{1}{2}\left(\mathcal{L}_{h X} g\right)(J Y, J Z),
$$

The tensors $\mathcal{C}$ and $\mathcal{C}^{\prime}$ are symmetric, semi-basics and

$$
\mathcal{C}(X, S)=\mathcal{C}^{\prime}(X, S)=0
$$

The $h$-torsion, $h v$-torsion and $v$-torsion of Cartan connection are given respectively by:

$$
T(h X, h Y)=\mathfrak{R}(X, Y), \quad T(h X, J Y)=\left(\mathcal{C}^{\prime}-F \mathcal{C}\right)(X, Y), \quad T(J X, J Y)=0 .
$$

Let $\stackrel{\circ}{R}$ and $\stackrel{\circ}{P}$ be the h- and hv-curvature tensors of Berwald connection and let $R$, $P$ and $Q$ be the h-, hv- and v-curvature tensors of Cartan connection.

Lemma 2.6. For Cartan connection, the following properties hold:

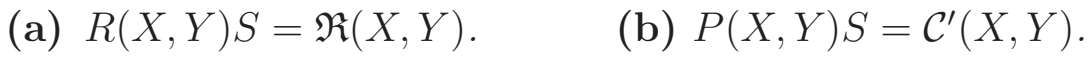

(c) $P(S, X) Y=P(X, S) Y=0$.

\section{Chern connection}

In this section, we prove the existence and uniqueness theorem of a remarkable connection: the Chern connection. We also give an explicit expressions for Chern connection.

We begin with some definitions quoted from [5].

Definition 3.1. A linear connection $\nabla$ on $T M$ is said to be regular if $\nabla J=0$ and the $\operatorname{map} \varphi: V(T M) \longrightarrow V(T M): X \longmapsto \nabla_{X} C$ is an isomorphism of $V(T M)$.

For a regular connection $\nabla$ on $T M$ there is associated a nonlinear connection $\Gamma$ on $M$ defied by $\Gamma=I-2 \varphi^{-1} \circ \nabla C ; \Gamma$ is said to be induced by $\nabla$.

Definition 3.2. A regular connection $\nabla$ on $T M$ is said to be reducible if $\nabla \Gamma=0$, where $\Gamma$ is the nonlinear connection induced by $\nabla$. 
Definition 3.3. A linear connection $\nabla$ on $T M$ is said to be almost-projectable if $\nabla J=0$ and $\nabla_{J X} C=J X$, for all $X \in T(T M)$. (an almost-projectable connection is necessairly regular).

If we replace the last axiom by the axiom $\nabla_{J X} J Y=J[J X, Y]$, the connection $\nabla$ is called normal almost-projectable.

The connection $\Gamma$ induced on $M$ by an almost-projectable (rep. normal almostprojectable) connection $\nabla$ on $T M$ will be called a projection (rep. normal projection) of $\nabla$. We also say that $\nabla$ projects (resp. projects normally) on $\Gamma$.

Definition 3.4. Let $\Gamma$ be a connection on $M$. The lift of $\Gamma$ is a reducible connection $\nabla$ on $T M$ which projects on $\Gamma$. The lift of $\Gamma$ is said to be normal if $\nabla$ is normal.

Lemma 3.5. For a reducible connection $\nabla$, we have $\nabla F=0$, where $F$ is the almostcomplex structure associated with the connection $\Gamma$ induced by $\nabla$.

Now, we are in a position to announce our fundamental result.

Theorem 3.6. For a Finsler manifold $(M, E)$ there exists a unique normal lift $\stackrel{*}{D}$ of Barthel connection $\Gamma=[J, S]$ such that:

(a) $\stackrel{*}{D}$ is horizontally metric: $\stackrel{*}{D}_{h X} g=0, \forall X \in \mathfrak{X}(T M)$.

(b) The classical torsion $\stackrel{*}{T}$ has the property that: $J \stackrel{*}{T}(h X, h Y)=0, \forall X, Y \in \mathfrak{X}(T M)$.

This connection is called Chern connection.

Proof. Firstly, we prove the uniqueness. Since $\stackrel{*}{D}$ is a normal lift of Barthel connection $\Gamma=[J, S]$, then

$$
\stackrel{*}{D}_{J X} J Y=J[J X, Y] \text {. }
$$

Also, by Lemma 3.5, we have

$$
\stackrel{*}{D} F=0 .
$$

Condition (a) implies that:

$$
\begin{aligned}
& h X . g(J Y, J Z)=g\left(\stackrel{*}{D}_{h X} J Y, J Z\right)+g\left(J Y, \stackrel{*}{D}_{h X} J Z\right), \\
& h Y . g(J Z, J X)=g\left(\stackrel{*}{D}_{h Y} J Z, J X\right)+g\left(J Z, \stackrel{*}{D}_{h Y} J X\right), \\
& h Z . g(J X, J Y)=g\left(\stackrel{*}{D}_{h Z} J X, J Y\right)+g\left(J X, \stackrel{*}{D}_{h Z} J Y\right) .
\end{aligned}
$$

By adding (3.3), (3.4) and subtracting (3.5), we get

$$
\begin{aligned}
h X . g(J Y, J Z)+ & h Y . g(J Z, J X)-h Y . g(J Z, J X)=g\left(\stackrel{*}{D}_{h X} J Y+\stackrel{*}{D}_{h Y} J X, J Z\right) \\
& +g\left(J Y, \stackrel{*}{D}_{h X} J Z-\stackrel{*}{D}_{h Z} J X\right)+g\left(\stackrel{*}{D}_{h Y} J Z-\stackrel{*}{D}_{h Z} J Y, J X\right) .
\end{aligned}
$$

Condition (b) together with $\stackrel{*}{D} J=0$ imply:

$$
\stackrel{*}{D}_{h X} J Y-\stackrel{*}{D}_{h Y} J X=J[h X, h Y] .
$$


From (3.6) and (3.7), we get

$$
\begin{array}{r}
g\left(2 \stackrel{*}{D}_{h X} J Y, J Z\right)=h X . g(J Y, J Z)+h Y . g(J Z, J X)-h Y . g(J Z, J X) \\
+g(J[h X, h Y], J Z)-g(J[h X, h Z], J Y)-g(J[h Y, h Z], J X)
\end{array}
$$

Since $\Omega(X, Y)=g(X, J Y)-g(J X, Y)$ and using $J \mathcal{C}^{\prime}=0$, then

$$
\frac{1}{2}\left(\mathcal{L}_{h X} g\right)(J Y, J Z)=\Omega\left(\mathcal{C}^{\prime}(X, Y), Z\right)=g\left(\mathcal{C}^{\prime}(X, Y), J Z\right)
$$

which is totally symmetric. Now,

$$
\begin{aligned}
g\left(2 \mathcal{C}^{\prime}(X, Y), J Z\right) & =h X . g(J Y, J Z)-g([h X, J Y], J Z)-g(J Y,[h X, J Z]), \\
g\left(2 \mathcal{C}^{\prime}(Y, Z), J X\right) & =h Y . g(J Z, J X)-g([h Y, J Z], J X)-g(J Z,[h Y, J X]), \\
-g\left(2 \mathcal{C}^{\prime}(Z, X), J Y\right) & =-h Z . g(J X, J Y)+g([h Z, J X], J Y)+g(J X,[h Z, J Y]) .
\end{aligned}
$$

By adding the above three equations, we get

$$
\begin{aligned}
g\left(2 \mathcal{C}^{\prime}(X, Y), J Z\right)= & h X . g(J Y, J Z)+h Y . g(J Z, J X)-h Z . g(J X, J Y) \\
& -g([h X, J Y]+[h Y, J X], J Z)+g([h Z, J X]-[h X, J Z], J Y) \\
& +g([h Z, J Y]-[h Y, J Z], J X)
\end{aligned}
$$

From (3.8) and (3.9), we have

$$
\begin{aligned}
g\left(2 \stackrel{*}{D}_{h X} J Y, J Z\right)= & g\left(2 \mathcal{C}^{\prime}(X, Y), J Z\right)+g([h X, J Y]+[h Y, J X]+J[h X, h Y], J Z) \\
& -g([h Z, J X]-[h X, J Z]+J[h X, h Z], J Y) \\
& -g([h Z, J Y]-[h Y, J Z]+J[h Y, h Z], J X) .
\end{aligned}
$$

Since the Barthel connection is torsion-free (Theorem 2.3), then

$$
0=t(X, Y)=v[J X, h Y]+v[h X, J Y]-J[h X, h Y]
$$

and so $J[h X, h Y]=v[J X, h Y]+v[h X, J Y]$. Hence, one can write:

$$
\begin{aligned}
{[h X, J Y]+[h Y, J X]+J[h X, h Y] } & =2 v[h X, h Y]+h[h X, J Y]+h[h Y, J X], \\
{[h Z, J X]-[h X, J Z]+J[h X, h Z] } & =h[J Z, J X]+h[h Z, J X], \\
{[h Z, J Y]-[h Y, J Z]+J[h Y, h Z] } & =h[h Z, J Y]+h[J Z, h Y] .
\end{aligned}
$$

The above relations and the the fact that $g(h X, J Y)=0$ enable us to write (3.10) in the form

$$
g\left(2 \stackrel{*}{D}_{h X} J Y, J Z\right)=g\left(2 \mathcal{C}^{\prime}(X, Y)+2 v[h X, J Y], J Z\right) .
$$

Hence,

$$
\stackrel{*}{D}_{h X} J Y=v[h X, J Y]+\mathcal{C}^{\prime}(X, Y) .
$$

Therefore, $\stackrel{*}{D}_{X} Y$ is uniquely determined by (3.1), (3.11) and (3.2). 
To prove the existence of $\stackrel{*}{D}$, let us define $\stackrel{*}{D}$ by the requirement that (3.1), (3.11) and (3.2) hold. Now, we have to prove that $\stackrel{*}{D}$ is a normal lift of $\Gamma=[J, S]$ (i.e., $\stackrel{*}{D} J=0$, $\left.\stackrel{*}{D} C=v, \stackrel{*}{D} \Gamma=0, \stackrel{*}{D}{ }_{J X} J Y=J[J X, Y]\right)$ and conditions (a) and (b) are satisfied.

- $\stackrel{*}{D} J=0$ : From (3.1), (3.2) and (3.11), we have

$$
\begin{aligned}
J \stackrel{*}{D}_{h X} Y & =J \stackrel{*}{D}_{h X} h Y+J \stackrel{*}{D}_{h X} v Y \\
& =J F \stackrel{*}{D}_{h X} J Y+\stackrel{*}{D}_{h X} J F Y \\
& =\left(J F v[h X, J Y]+v \mathcal{C}^{\prime}(X, Y)\right)+\left(J v[h X, v Y]+J \mathcal{C}^{\prime}(X, F Y)\right) \\
& =v[h X, J Y]+\mathcal{C}^{\prime}(X, Y), \text { since } \mathcal{C}^{\prime} \text { is semi basic and } J v=0 \\
& =\stackrel{*}{D}_{h X} J Y .
\end{aligned}
$$

Similarly, one can show that $J^{\stackrel{*}{D}}{ }_{v X} Y=\stackrel{*}{D}_{v X} J Y$.

- ${ }^{*} C=v$ : From (3.11), (2.7) and (2.3), we get

$$
\stackrel{*}{D}_{h X} C=\stackrel{*}{D}_{h X} J S=v[h X, J S]+\mathcal{C}^{\prime}(X, S)=-v[C, h X]=-v h[C, X]=0 .
$$

On the other hand, from (3.1) and (2.2), we obtain $\stackrel{*}{D}_{J X} C=\stackrel{*}{D}_{J X} J S=J[J X, S]=J X$.

- $\stackrel{*}{D} \Gamma=0$ or, equivalently, $\stackrel{*}{D} h=0$ :

$$
\begin{aligned}
h \stackrel{*}{D}_{h X} Y & =h \stackrel{*}{D}_{h X} h Y+h \stackrel{*}{D}_{h X} v Y=h \stackrel{*}{D}_{h X} h Y, \text { since } \stackrel{*}{D}_{h X} v Y \text { is vertical by (3.11) } \\
& =h \stackrel{*}{D}_{h X} F J Y=h F \stackrel{*}{D}_{h X} J Y, \text { by (3.2) } \\
& =h F v[h X, J Y]+h F \mathcal{C}^{\prime}(X, Y) \\
& =F v^{2}[h X, J Y]+F v \mathcal{C}^{\prime}(X, Y) \\
& =F v[h X, J Y]+F \mathcal{C}^{\prime}(X, Y)=F D_{h X} J Y=\stackrel{*}{D}_{h X} h Y .
\end{aligned}
$$

Similarly, $\stackrel{*}{D}_{J X} h Y=h \stackrel{*}{D}_{J X} Y$.

- ${ }^{*}$ is h-metrical: As $g(J X, J Y)=g(h X, h Y)$ and $g(h X, J Y)=0$, it suffices to prove that $\left.\stackrel{*}{D}_{h X} g\right)(J Y, J Z)=0$. By (3.1) $)$ (3.2) and (3.11) , we have

$$
\begin{aligned}
\left(\mathcal{*}_{h X} g\right)(J Y, J Z)= & h X . g(J Y, J Z)-g\left({ }^{*}{ }_{h X} J Y, J Z\right)-g\left(J Y,{ }^{*}\right. \\
= & h X . g(J Y, J Z)-g(v[h X, J Y], J Z)-g\left(\mathcal{C}^{\prime}(X, Y), J Z\right) \\
& -g(J Y, v[h X, J Z])-g\left(J Y, \mathcal{C}^{\prime}(X, Z)\right) \\
= & h X . g(J Y, J Z)-g(v[h X, J Y], J Z)-g(J Y, v[h X, J Z]) \\
& -2 \mathcal{C}_{b}^{\prime}(X, Y, Z)=0 .
\end{aligned}
$$

- $J \mathbf{T}(h X, h Y)=0$ : By (3.11) and the symmetry of $\mathcal{C}^{\prime}$, we have

$$
\begin{aligned}
J_{T}^{*}(h X, h Y) & =\stackrel{*}{D}_{h X} h Y-\stackrel{*}{D}_{h Y} h X-J[h X, h Y] \\
& =\stackrel{*}{D}_{h X} J Y-\stackrel{*}{D}_{h Y} J X-J[h X, h Y] \\
& =v[h X, J Y]+\mathcal{C}^{\prime}(X, Y)-v[h Y, J X]-\mathcal{C}^{\prime}(Y, X)-J[h X, h Y] \\
& =t(X, Y)=0 .
\end{aligned}
$$

This completes the proof. 
Corollary 3.7. The Chern connection $\stackrel{*}{D}$ is completely determined by:

(a) $\stackrel{*}{D}_{J X} J Y=J[J X, Y]=\stackrel{\circ}{D}_{J X} J Y$.

(b) $\stackrel{*}{D}_{h X} J Y=v[h X, J Y]+\mathcal{C}^{\prime}(X, Y)=D_{h X} J Y$.

(c) $\stackrel{*}{D} F=0$.

\section{Torsion and curvature tensors}

In this section, we study the torsion and curvature tensors of Chern connection. We also derive the Bianchi identities and obtain some properties of the curvature tensors. We start with the following lemma which will be useful for subsequent use.

Lemma 4.1. For all $X, Y \in \mathfrak{X}(T M)$, we have

(a) $[J X, J Y]=J\left(\stackrel{*}{D}_{J X} Y-\stackrel{*}{D}_{J Y} X\right)$.

(b) $[h X, J Y]=J\left(\stackrel{*}{D}_{h X}\right) Y-h\left(\stackrel{*}{D}_{J Y} X\right)-\mathcal{C}^{\prime}(X, Y)$.

(c) $[h X, h Y]=h\left(\stackrel{*}{D}_{h X} Y-\stackrel{*}{D}_{h Y} X\right)-\mathfrak{R}(X, Y)$.

Proof.

(a) By Corollary 3.7 and the fact that $[J, J]=0$ (2.1), we get

$$
J\left(\stackrel{*}{D}_{J X} Y-\stackrel{*}{D}_{J Y} X\right)=\stackrel{*}{D}_{J X} J Y-\stackrel{*}{D}_{J Y} J X=J[J X, Y]-J[J Y, X]=[J X, J Y] .
$$

(b) By Corollary 3.7 and the identity $h[J Y, X]=h[J Y, h X]$, we obtain

$$
\begin{aligned}
J\left(\stackrel{*}{D}_{h X} Y\right)-h\left(\stackrel{*}{D}_{J Y} X\right) & =\stackrel{*}{D}_{h X} J Y-\stackrel{*}{D}_{J Y} h X \\
& =v[h X, J Y]+\mathcal{C}^{\prime}(X, Y)-h[J Y, X] \\
& =v[h X, J Y]-h[J Y, X]+\mathcal{C}^{\prime}(X, Y) \\
& =v[h X, J Y]+h[h X, J Y]+\mathcal{C}^{\prime}(X, Y) \\
& =[h X, J Y]+\mathcal{C}^{\prime}(X, Y) .
\end{aligned}
$$

(c) Again by Corollary 3.7 and the symmetry property of $\mathcal{C}^{\prime}$, we have

$$
\begin{aligned}
h\left(\stackrel{*}{D}_{h X} Y-\stackrel{*}{D}_{h Y} X\right) & =\stackrel{*}{D}_{h X} h Y-\stackrel{*}{D}_{h Y} h X \\
& =F v[h X, J Y]+F \mathcal{C}^{\prime}(X, Y)-F v[h Y, J X]-F \mathcal{C}^{\prime}(Y, X) \\
& =F v[h X, J Y]+F v[J X, h Y] .
\end{aligned}
$$

As the torsion of $\Gamma$ vanishes, then $0=t(X, Y)=v[J X, h Y]+v[h X, J Y]-J[h X, h Y]$, from which $F v[J X, h Y]+F v[h X, J Y]=F J[h X, h Y]=h[h X, h Y]$. Consequently,

$$
h\left(\stackrel{*}{D}_{h X} Y-\stackrel{*}{D}_{h Y} X\right)=h[h X, h Y]=[h X, h Y]-v[h X, h Y]=[h X, h Y]+\mathfrak{R}(X, Y),
$$

where we have used the identity $\mathfrak{R}(X, Y)=-v[h X, h Y][10]$. 
Remark 4.2. The last identity of Lemma 4.1 retrieves a result of [10]: A necessary and sufficient condition for the horizontal distribution to be completely integrable is that $\mathfrak{R}$ vanishes.

Proposition 4.3. The h-torsion, hv-torsion and $v$-torsion of Chern connection $\stackrel{*}{D}$ are given by:
(a) $\stackrel{*}{T}(h X, h Y)=\mathfrak{R}(X, Y)$.
(b) $\stackrel{*}{T}(h X, J Y)=\mathcal{C}^{\prime}(X, Y)$.
(c) $\stackrel{*}{T}(J X, J Y)=0$.

Proof.

(a) Follows directly from the definition of ${ }^{*}(h X, h Y)$ and Lemma 4.1 (c).

(b) By Corollary 3.7 and using the property that $h[J X, v Y]=0$, we get

$$
\begin{aligned}
\stackrel{*}{T}(h X, J Y) & =\stackrel{*}{D}_{h X} J Y-\stackrel{*}{D}_{J Y} h X-[h X, J Y] \\
& =v[h X, J Y]+\mathcal{C}^{\prime}(X, Y)-h[J Y, X]-[h X, J Y] \\
& =v[h X, J Y]+\mathcal{C}^{\prime}(X, Y)-h[J Y, h X]-(h[h X, J Y]+v[h X, J Y]) \\
& =\mathcal{C}^{\prime}(X, Y) .
\end{aligned}
$$

(c) Is obvious.

As $\stackrel{*}{D} F=0$, the (classical) curvature tensor $K$ of Chern connection is completely determined by the three curvature tensors: $h$-curvature $\stackrel{*}{R}, h v$-curvature $\stackrel{*}{P}$ and $v$ curvature ${ }^{*}$ defined respectively by:

$$
\begin{aligned}
& \stackrel{*}{R}(X, Y) Z=K(h X, h Y) J Z, \\
& \stackrel{*}{P}(X, Y) Z=K(h X, J Y) J Z, \\
& \stackrel{*}{Q}(X, Y) Z=K(J X, J Y J) Z .
\end{aligned}
$$

Proposition 4.4. The h-curvature $\stackrel{*}{R}$, hv-curvature $\stackrel{*}{P}$ and $v$-curvature $\stackrel{*}{Q}$ of the Chern connection are given by:

(a) $\stackrel{*}{R}(X, Y) Z=R(X, Y) Z-\mathcal{C}(F \Re(X, Y), Z)$.

(b) $\stackrel{*}{P}(X, Y) Z=\stackrel{\circ}{P}(X, Y) Z-\left(\stackrel{*}{D}_{J Y} \mathcal{C}^{\prime}\right)(X, Z)$.

(c) $\stackrel{*}{Q}(X, Y) Z=0$.

Proof. We prove (a) only. The other expressions can be proved similarly. As $\stackrel{*}{D}_{h X} J Y=D_{h X} J Y$ (Corollary $\left.3.7(\mathrm{~b})\right)$, we have

$$
\begin{aligned}
\stackrel{*}{R}(X, Y) Z & =\stackrel{*}{D}_{h X} \stackrel{*}{D}_{h Y} J Z-\stackrel{*}{D}_{h Y} \stackrel{*}{D}_{h X} J Z-\stackrel{*}{D}_{[h X, h Y]} J Z \\
& =D_{h X} D_{h Y} J Z-D_{h Y} D_{h X} J Z-\stackrel{*}{D}_{[h X, h Y]} J Z \\
& =R(X, Y) Z+D_{[h X, h Y]} J Z-\stackrel{*}{D}_{[h X, h Y]} J Z \\
& =R(X, Y) Z+D_{J F[h X, h Y]} J Z-\stackrel{*}{D}_{J F[h X, h Y]} J Z
\end{aligned}
$$


By (2.6) and Corollary 3.7, the last equation takes the form

$$
\begin{aligned}
\stackrel{*}{R}(X, Y) Z & =R(X, Y) Z+\mathcal{C}(F[h X, h Y], Z) \\
& =R(X, Y) Z-\mathcal{C}(F \Re(X, Y), Z),
\end{aligned}
$$

where we have used the identity $\mathfrak{R}(X, Y)=-v[h X, h Y]$ and the fact that $C$ is semibasic.

Proposition 4.5. The h-curvature $\stackrel{*}{R}$ and hv-curvature $\stackrel{*}{P}$ of Chern connection have the following properties:

(a) $\stackrel{*}{R}(X, Y) S=\mathfrak{R}(X, Y)$.

(b) $\stackrel{*}{P}(X, Y) S=\stackrel{*}{P}(S, Y) X=\mathcal{C}^{\prime}(X, Y)$.

(c) $\stackrel{*}{P}(X, S) Z=0$.

Proof.

(a) Follows from Proposition 4.4, Lemma 2.6 and (2.7).

(b) By Proposition 4.4, (2.2), the properties of $\mathcal{C}^{\prime}$ and the properties of $\stackrel{\circ}{P}$, we get

$$
\begin{aligned}
\stackrel{*}{P}(X, Y) S & =-\left(\stackrel{*}{D}_{J Y} \mathcal{C}^{\prime}\right)(X, S)=-\left(\stackrel{\circ}{D}_{J Y} \mathcal{C}^{\prime}\right)(X, S)=\mathcal{C}^{\prime}\left(X, \stackrel{\circ}{D}_{J Y} S\right)=\mathcal{C}^{\prime}(X, F J[J Y, S]) \\
& =\mathcal{C}^{\prime}(X, F J Y)=\mathcal{C}^{\prime}(X, Y) .
\end{aligned}
$$

(c) can be proved similarly.

To study the Bianchi identities for Chern connection, let us first write the Bianchi identities for an arbitrary connection $\nabla$.

Lemma 4.6. Let $\nabla$ be a linear connection on $M$ with torsion tensor $\boldsymbol{T}$ and curvature tensor $\boldsymbol{K}$. For every $X, Y, Z \in \mathfrak{X}(M)$, we have:

(I) $\mathfrak{S}_{X, Y, Z}\{\boldsymbol{K}(X, Y) Z\}=\mathfrak{S}_{X, Y, Z}\left\{\boldsymbol{T}(\boldsymbol{T}(X, Y), Z)+\left(\nabla_{X} T\right)(Y, Z)\right\}$,

(II) $\mathfrak{S}_{X, Y, Z}\left\{\boldsymbol{K}(\boldsymbol{T}(X, Y), Z)+\left(\nabla_{X} \boldsymbol{K}\right)(Y, Z)\right\}=0$,

where the symbol $\mathfrak{S}_{X, Y, Z}$ denotes cyclic sum over $X, Y$ and $Z$.

Applying the identities (I) and (II) on Chern connection, for different triples of vector fields $(h X, h Y, h Z),(h X, h Y, J Z), \ldots$, we obtain many identities. Here, we give only the most important of these identities.

Proposition 4.7. The first Bianchi identity for Chern connection yields:

(a) $\mathfrak{S}_{X, Y, Z}\{\stackrel{*}{R}(X, Y) Z\}=0$.

(b) $\mathfrak{S}_{X, Y, Z}\left\{\left(\stackrel{*}{D}_{h X} \mathfrak{R}\right)(Y, Z)\right\}=\mathfrak{S}_{X, Y, Z}\left\{\mathcal{C}^{\prime}(F \mathfrak{R}(X, Y), Z)\right\}$.

(c) $\stackrel{*}{P}(X, Y) Z=\stackrel{*}{P}(Z, Y) X$.

(d) $\stackrel{*}{P}(X, Y) Z-\stackrel{*}{P}(X, Z) Y=\left(\stackrel{*}{D}_{J Z} \mathcal{C}^{\prime}\right)(X, Y)-\left(\stackrel{*}{D}_{J Y} \mathcal{C}^{\prime}\right)(X, Z)$. 
The second Bianchi identity for Chern connection yields:

(e) $\mathfrak{S}_{X, Y, Z}\left\{\left(\stackrel{*}{D_{h X}} \stackrel{*}{R}\right)(Y, Z)\right\}=\mathfrak{S}_{X, Y, Z}\{\stackrel{*}{P}(X, F \mathfrak{R}(Y, Z))\}$.

(f) $\left(\stackrel{*}{D}{ }_{h X} \stackrel{*}{P}\right)(Y, Z)-\left(\stackrel{*}{D_{h Y}} \stackrel{*}{P}\right)(X, Z)+\left(\stackrel{*}{D}_{J Z} \stackrel{*}{R}\right)(X, Y)=\stackrel{*}{P}\left(X, F \mathcal{C}^{\prime}(Y, Z)\right)$ $-\stackrel{*}{P}\left(Y, F \mathcal{C}^{\prime}(X, Z)\right)$.

(g) $\left(\stackrel{*}{D}_{J Y} \stackrel{*}{P}\right)(X, Z)=\left(\stackrel{*}{D}_{J Z} \stackrel{*}{P}\right)(X, Y)$.

Corollary 4.8. The h-curvature $\stackrel{*}{R}$ and the hv-curvature $\stackrel{*}{P}$ satisfy:
(a) $\stackrel{*}{D_{C}} \stackrel{*}{R}=0$,
(b) $\stackrel{*}{D} \stackrel{*}{P}=0$,
(c) $\stackrel{*}{P}$ is totally symmetric if $\stackrel{*}{D}{ }_{J Z} \mathcal{C}^{\prime}=0$.

Proposition 4.9. The h-curvature $\stackrel{*}{R}$ has the following properties:

(a) $\stackrel{*}{R}(X, Y, Z, W)=-\stackrel{*}{R}(Y, X, Z, W)$,

(b) $\stackrel{*}{R}(X, Y, Z, W)+\stackrel{*}{R}(Y, Z, X, W)+\stackrel{*}{R}(Z, X, Y, W)=0$,

Moreover, if $\mathfrak{R}=0$, we have

(c) $\stackrel{*}{R}(X, Y, Z, W)=-\stackrel{*}{R}(X, Y, W, Z)$,

(d) $\stackrel{*}{R}(X, Y, Z, W)=\stackrel{*}{R}(Z, W, X, Y)$,

where $\stackrel{*}{R}(X, Y, Z, W):=g(\stackrel{*}{R}(X, Y) Z, J W)$.

Proof. (a) is clear.

(b) We have

$$
\begin{aligned}
\stackrel{*}{R}(X, Y, Z, W)+\stackrel{*}{R}(Y, Z, X, W)+\stackrel{*}{R}(Z, X, Y, W) \\
\quad=g(\stackrel{*}{R}(X, Y) Z, J W)+g(\stackrel{*}{R}(Y, Z) X, J W)+g(\stackrel{*}{R}(Z, X) Y, J W) \\
\quad=g(\stackrel{*}{R}(X, Y) Z+\stackrel{*}{R}(Y, Z) X+\stackrel{*}{R}(Z, X) Y, J W)=0, \text { by Proposition } 4.7 .
\end{aligned}
$$

(c) By Theorem 3.6, we have

$$
h X . g(J Y, J Z)=g\left(\stackrel{*}{D}_{h X} J Y, J Z\right)+g\left(J Y, \stackrel{*}{D}_{h X} J Z\right) .
$$

Then, we can write

$$
\begin{gathered}
h W \cdot(h X . g(J Y, J Z))=g\left(\stackrel{*}{D}_{h W} \stackrel{*}{D}_{h X} J Y, J Z\right)+g\left(\stackrel{*}{D}_{h X} J Y, \stackrel{*}{D_{h W}} J Z\right) \\
+g\left(\stackrel{*}{D}_{h W} J Y, \stackrel{*}{D}_{h X} J Z\right)+g\left(J Y, \stackrel{*}{D}_{h W} \stackrel{*}{D}_{h X} J Z\right) .
\end{gathered}
$$

Interchanging $X$ and $W$, we get

$$
\begin{gathered}
h X .(h W . g(J Y, J Z))=g\left(\stackrel{*}{D}_{h X}^{*} \stackrel{*}{D}_{h W} J Y, J Z\right)+g\left(\stackrel{*}{D}_{h W} J Y,{ }^{*}{ }_{h X}^{*} J Z\right) \\
+g\left(*_{h X}^{*} J Y,{ }_{h W}^{*} J Z\right)+g\left(J Y,\left(*_{h X}^{*}{ }_{h W}^{*} J Z\right)\right) .
\end{gathered}
$$

Using the above two equations, we obtain

$$
\begin{aligned}
{[h W, h X] . g(J Y, J Z)=} & g\left(\left(\stackrel{*}{D}_{h W} \stackrel{*}{D}_{h X}-\stackrel{*}{D}_{h X} \stackrel{*}{D}_{h W}\right) J Y, J Z\right) \\
& +g\left(J Y,\left(\stackrel{*}{D}_{h W} *_{h X}^{*}-*_{h X}^{*} *_{h W}^{*}\right) J Z\right) .
\end{aligned}
$$

If $\mathfrak{R}=0$, then the horizontal distribution is completely integrable. Consequently, $[h W, h X]$ is horizontal and so $\stackrel{*}{D}_{[h W, h X]} g=0$. Hence, we have 


$$
[h W, h X] . g(J Y, J Z)=g\left(\stackrel{*}{D}_{[h W, h X]} J Y, J Z\right)+g\left(J Y, \stackrel{*}{D}_{[h W, h X]} J Z\right) .
$$

Comparing the above two equations, we get

From which,

$$
\begin{aligned}
& g\left(\left(\stackrel{*}{D}_{h W} \stackrel{*}{D}_{h X}-\stackrel{*}{D}_{h X} \stackrel{*}{D}_{h W}-\stackrel{*}{D}_{[h W, h X]}\right) J Y, J Z\right) \\
& +g\left(J Y,\left(\stackrel{*}{D}_{h W} \stackrel{*}{D}_{h X}-\stackrel{*}{D}_{h X} \stackrel{*}{D}_{h W}-\stackrel{*}{D}_{[h W, h X]}\right) J Z\right)=0 .
\end{aligned}
$$

$$
\stackrel{*}{R}(W, X, Y, Z)=-\stackrel{*}{R}(W, X, Z, Y) .
$$

(d) Using (a), (b) and (c), since $\mathfrak{R}=0$, we have

$$
\begin{aligned}
& \stackrel{*}{R}(X, Y, Z, W)=-\stackrel{*}{R}(Y, X, Z, W)=\stackrel{*}{R}(X, Z, Y, W)+\stackrel{*}{R}(Z, Y, X, W), \\
& \stackrel{*}{R}(X, Y, Z, W)=-\stackrel{*}{R}(X, Y, W, Z)=\stackrel{*}{R}(Y, W, X, Z)+\stackrel{*}{R}(W, X, Y, Z) .
\end{aligned}
$$

Adding the above two equation, we get

$$
\begin{aligned}
2 \stackrel{*}{R}(X, Y, Z, W)= & \stackrel{*}{R}(X, Z, Y, W)+\stackrel{*}{R}(Z, Y, X, W) \\
& +\stackrel{*}{R}(Y, W, X, Z)+\stackrel{*}{R}(W, X, Y, Z) .
\end{aligned}
$$

Similarly,

$$
\begin{aligned}
2 \stackrel{*}{R}(Z, W, X, Y)= & \stackrel{*}{R}(Z, X, W, Y)+\stackrel{*}{R}(X, W, Z, Y) \\
& +\stackrel{*}{R}(W, Y, Z, X)+\stackrel{*}{R}(Y, Z, W, X) .
\end{aligned}
$$

Comparing (4.1) and (4.2), we get $\stackrel{*}{R}(X, Y, Z, W)=\stackrel{*}{R}(Z, W, X, Y)$.

Remark 4.10. It is to be noted that if $\mathfrak{R}$ vanishes, we get some interesting results:

- $\stackrel{*}{R}=R$ (Proposition 4.4 (a)) and also $\mathfrak{S}_{X, Y, Z}\{R(X, Y) Z\}=0$.

- $\mathfrak{S}_{X, Y, Z}\left\{\left(\stackrel{*}{D}_{h X} \stackrel{*}{R}\right)(Y, Z)\right\}=0$ (Proposition $\left.4.7(\mathbf{e})\right)$.

- The properties (c) and (d) in Proposition 4.9 hold.

The above properties are very similar to the properties of the Riemannian curvature. The reason lies in the condition $\mathfrak{R}=0$ which is stronger than the condition (b) of Theorem 3.6. More precisely, the condition ${ }^{*}(h X, h Y)=\Omega(X, Y)=0$ is stronger than the condition $J \stackrel{*}{T}(h X, h Y)=0$.

\section{Appendix: Intrinsic Comparison}

The following table gives a concise comparison concerning Berwald, Cartan and Chern connections as well as the fundamental geometric objects associated with them. 
Table 1: Intrinsic Comparison

\begin{tabular}{|c|c|c|c|}
\hline Connection & Berwald: ${ }^{\circ}$ & Cartan: $D$ & Chern: ${ }^{*}$ \\
\hline Expression & $\begin{array}{c}\stackrel{\circ}{D}_{J X} J Y=J[J X, Y] \\
\stackrel{\circ}{D}_{h X} J Y=v[h X, J Y] \\
\stackrel{\circ}{D} F=0\end{array}$ & $\begin{array}{c}D_{J X} J Y=\stackrel{\circ}{D}_{J X} J Y+\mathcal{C}(X, Y) \\
D_{h X} J Y=\stackrel{\circ}{D}_{h X} J Y+\mathcal{C}^{\prime}(X, Y) \\
D F=0\end{array}$ & $\begin{array}{l}\stackrel{*}{D}_{J X} J Y=\stackrel{\circ}{D}_{J X} J Y \\
\stackrel{*}{D}_{h X} J Y=D_{h X} J Y \\
\quad \stackrel{*}{D F}=0\end{array}$ \\
\hline $\begin{array}{l}h \text {-torsion } \\
h \text { v-torsion } \\
v \text {-torsion }\end{array}$ & $\begin{array}{l}\Re \\
0 \\
0\end{array}$ & $\begin{array}{c}\mathfrak{R} \\
\mathcal{C}^{\prime}-F \mathcal{C} \\
0\end{array}$ & $\begin{array}{l}\mathfrak{R} \\
\mathcal{C}^{\prime} \\
0\end{array}$ \\
\hline$h$-curvature & $\stackrel{\circ}{R}(X, Y) Z=\left(\stackrel{\circ}{D}_{J Z} \mathfrak{R}\right)(X, Y)$ & $\begin{array}{c}R(X, Y) Z=R(X, Y) Z+\left(D_{h X} \mathcal{C}^{\prime}\right)(Y, Z) \\
-\left(D_{h Y} \mathcal{C}^{\prime}\right)(X, Z)+\mathcal{C}^{\prime}\left(F \mathcal{C}^{\prime}(X, Z), Y\right) \\
\mathcal{C}^{\prime}\left(F \mathcal{C}^{\prime}(Y)\right.\end{array}$ & $\begin{array}{l}\stackrel{*}{R}(X, Y) Z=R(X, Y) Z \\
\quad-\mathcal{C}(F \Re(X, Y), Z)\end{array}$ \\
\hline$h v$-curvature & $\begin{array}{c}\stackrel{\circ}{P}(X, Y) Z=v[h X, J[J Y, Z]] \\
-\mathrm{J}[\mathrm{JY}, \mathrm{F}[\mathrm{hX}, \mathrm{JZ}]]-\mathrm{v}[\mathrm{h}[\mathrm{hX}, \mathrm{JY}], \mathrm{JZ}] \\
\text {-J[v[hX,JY],Z] }\end{array}$ & $\begin{array}{c}P(X, Y) Z=\stackrel{\circ}{P}(X, Y) Z+\left(D_{h X} \mathcal{C}\right)(Y, Z) \\
-\left(D_{J Y} \mathcal{C}^{\prime}\right)(X, Z)+\mathcal{C}\left(F \mathcal{C}^{\prime}(X, Z), Y\right) \\
+\mathcal{C}\left(F \mathcal{C}^{\prime}(X, Y), Z\right)-\mathcal{C}^{\prime}(F \mathcal{C}(Y, Z), X) \\
-\mathcal{C}^{\prime}(F \mathcal{C}(X, Y), Z)\end{array}$ & $\begin{array}{c}\stackrel{*}{P}(X, Y) Z=\stackrel{\circ}{P}(X, Y) Z \\
\quad-\left(\stackrel{*}{D}_{J Y} \mathcal{C}^{\prime}\right)(X, Z)\end{array}$ \\
\hline$v$-curvature & 0 & $\begin{array}{c}Q(X, Y) Z=\mathcal{C}(F \mathcal{C}(X, Z), Y) \\
-\mathcal{C}(F \mathcal{C}(Y, Z), X)\end{array}$ & 0 \\
\hline $\begin{array}{l}v \text {-metricity } \\
h \text {-metricity }\end{array}$ & $\begin{array}{l}\text { not } v \text {-metrical } \\
\text { not } h \text {-metrical }\end{array}$ & $\begin{array}{l}v \text {-metrical } \\
h \text {-metrical }\end{array}$ & $\begin{array}{c}\text { not } v \text {-metrical } \\
h \text {-metrical }\end{array}$ \\
\hline
\end{tabular}




\section{References}

[1] H. Akbar-Zadeh, Initiation to global Finsler geometry, Elsevier, 2006.

[2] P. Dazord, Propriétés globales des géodésiques des espaces de Finsler, Thèse d'Etat, (575) Publ. Dept. Math., Univ. Lyon, 1969.

[3] A. Frölicher and A. Nijenhuis, Theory of vector-valued differential forms, I, Ann. Proc. Kon. Ned. Akad., A, 59 (1956), 338-359.

[4] J. Grifone, Structure presque-tangente et connexions, I, Ann. Inst. Fourier, Grenoble, 22, 1 (1972), 287-334.

[5] J. Grifone, Structure presque-tangente et connexions, II, Ann. Inst. Fourier, Grenoble, 22, 3 (1972), 291-338.

[6] J. Klein and A. Voutier, Formes extérieures génératrices de sprays, Ann. Inst. Fourier, Grenoble, 18, 1 (1968), 241-260.

[7] M. Matsumoto, The theory of Finsler connections, Publication of the study group of geometry, Vol. 5, Dept. Math., Okayama Univ., 1970.

[8] J. Szilasi and C. Vincze, A new look at Finsler connections and special Finsler manifolds, Acta Math. Acad. Paedagog. Nyházi., N. S., 16 (2000), 33-63.

[9] A. A. Tamim, General theory of Finsler spaces with applications to Randers spaces, Ph. D. Thesis, Cairo Univ., 1991.

[10] Nabil L. Youssef, Distribution de nullité du tensor de courbure d'une connexion, C. R. Acad. Sci. Paris, Sér. A, 290 (1980), 653-656.

[11] Nabil L. Youssef, S. H. Abed and A. Soleiman, Cartan and Berwald connections in the pullback formalism, Algebras, Groups and Geometries, 25, 4 (2008), 363-386. arXiv: 0707.1320 [math. DG].

[12] Nabil L. Youssef, S. H. Abed and A. Soleiman, A global approach to the theory of connections in Finsler geometry, Tensor, N. S., 71, 3 (2009), 187-208. arXiv: 0801.3220 [math.DG]. 